\title{
Sterilisation and single-use of endodontic files - a short report
}

SADJ May 2020, Vol. 75 No. 4 p 189 - p 191

GD Buchanan', MY Gamieldien², S Tredoux ${ }^{3}$, N Warren ${ }^{4}$

\section{ABSTRACT}

\section{Introduction}

In many countries, endodontic files continue to be sterilised and reused in multiple clinical cases as the alternative of single-use of these instruments is avoided due to financial reasons.

\section{Methods}

A survey was performed including South African general dental practitioners and specialist prosthodontists to determine the current endodontic file sterilisation methods in place and the adoption rate of single-use protocols of both hand and engine-driven instruments.

\section{Results}

The majority of respondents $(76.6 \%, n=141 / 184)$ indicated autoclaving endodontic files prior to reuse. Almost a quarter of the respondents $(23.4 \%,(n=43 / 184)$ used only cold sterilisation.

Some respondents $(2,8 \%, n=5 / 181)$ indicated not sterilising their files at all. A low number of respondents had adopted the single-use of endodontic hand files (10.9\%, $n=20 / 184)$. Engine-driven files were used only once and discarded by $18.5 \%(n=34 / 184)$ of respondents.

\section{Conclusion}

It appears that, at times, acceptable infection prevention and control practices regarding the sterilisation these

\section{Author affiliations:}

1. Glynn D Buchanan: BChD, PDD (Endo), MSc (Dent), Department of Odontology, School of Dentistry, University of Pretoria, Pretoria, South Africa.

ORCID Number: 0000-0003-2957-166X

2. Mohamed Y Gamieldien: BChD, PGDipDent (Oral Surg), MSc (Anat), Department of Maxillofacial and Oral Surgery, School of Dentistry, University of Pretoria, Pretoria, South Africa.

3. Sheree Tredoux: $B C h D, P D D$ (Endo), Department of Odontology, School of Dentistry, University of Pretoria, Pretoria, South Africa.

4. Nichola Warren: BChD, PGDipDent (Endo), MSc (Odont), Department of Odontology, School of Dentistry, University of Pretoria, Pretoria, South Africa.

Corresponding author: Glynn D Buchanan

Oral and Dental Hospital, 31 Bophelo Road, Prinshof Campus, Riviera,

Pretoria, 0002, South Africa.

Email: glynn.buchanan@up.ac.za

Author contributions:

All authors have contributed significantly and are in agreement with the manuscript.

1. Glynn D Buchanan: Conceived the study, wrote, reviewed and edited the manuscript - $40 \%$

2. Mohamed Y Gamieldien: Conceived the study and wrote, reviewed and edited the manuscript - $30 \%$

3. Sheree Tredoux: Conceived the study and wrote, reviewed and edited the manuscript - $20 \%$

4. Nichola Warren: Conceived the study and wrote, reviewed and edited the manuscript - $10 \%$ instruments are not necessarily being adhered to. The adoption of single-use protocols of endodontic files in a South African dental setting is higher than previously reported.

\section{Keywords}

Endodontic files, single-use, sterilisation, survey.

\section{INTRODUCTION}

In modern dental practice, both hand and engine-driven endodontic files are used for cleaning and shaping procedures of root canal treatment. During this process, these instruments become contaminated with microorganisms and appropriate cross-contamination and sterilisation procedures are necessary if endodontic files are to be reused on multiple cases. ${ }^{1}$

Whilst it is common practice to reprocess many dental instruments, the intricate designs of some make effective cleaning and sterilising a challenge. For this reason several instruments are considered disposable or singleuse items. ${ }^{2}$

Previous authors have supported the recommendation for the single-use of both hand and rotary endodontic files due to the inability to adequately reprocess these instruments, ${ }^{2-4}$ however this view has been debated. ${ }^{5}$ Despite these findings, dentists in many countries continue to reprocess and reuse rotary and hand files in multiple cases following sterilisation procedures.

It has been previously reported that infection prevention and control practices in a South African dental setting may at times be inadequate. ${ }^{6,7}$ The sterilisation methods used by South African dental practitioners specifically for the reprocessing of endodontic files is currently unclear.

Furthermore, the proportion of South African practitioners who have adopted single-use protocols, pertaining only to endodontic hand files, has only been previously reported on in one major city. ${ }^{8}$

This study aimed to determine the sterilisation methods used by South African dental practitioners in the reprocessing of both engine-driven as well as endodontic hand files, and to quantify the proportion of practitioners who practice single-use of these instruments.

\section{MATERIALS AND METHODS}

A cross-sectional, observational study was conducted. A multiple-choice survey was designed using an online program (Qualtrics, Provo, Utah) and electronically mailed 
to 61 members of the Academy of Prosthodontics, South Africa (APSA) and 3191 South African General Dental Practitioners (GDPs) registered in the South African Dental Association (SADA) database.

The survey was circulated on social media platforms to increase visibility to the target groups. A quantitative design consisting of questions with multiple-choice answers was completed by participants. The questions included:

- Demographic information.

- Sterilisation methods used for endodontic instruments.

- Sterilisation conducted prior to first use of new files.

- Sterilisation conducted prior to re-use.

- Single-use of endodontic hand files.

- Single-use of engine-driven files.

Data was collected and exported as comma-separated values (CSV file format) for evaluation in Microsoft Excel 2016, analysed and expressed as simple percentages of the total number of respondents.

Only private practice GDPs and SPs routinely performing endodontic treatment were included in this investigation. GDPs and SPs not engaged in the clinical practice of endodontics and those employed outside of a private practice setting, such as community-service (a compulsory internship year in South Africa), public-sector dentists and full-time academics were excluded.

The research proposal for this study was approved by the Faculty of Health Sciences Research Ethics Committee, University of Pretoria (Protocol number 331/2018).

\section{RESULTS}

An electronic mail containing a link to the survey was sent to both APSA members $(n=61)$ and SADA members $(n=3191)$. In total, 215 responses were returned by the cut-off date. The overall response rate was $6.6 \%$ of the total number surveyed $(n=215 / 3252)$.

Almost ten percent of the total respondents $(9.7 \%$, $n=21 / 215)$ indicated not performing private practice endodontic treatment and were excluded. Another respondent provided irrational answers to several questions and was therefore ruled out for inclusion. Analysis was subsequently performed on the valid responses provided by the remaining 193 participants $(89.8 \%$ of total respondents).

\section{Demographics}

Of the 193 participants, $46.1 \% \quad(n=89)$ were male and $53.9 \%(n=104)$ were female. Eleven participants $(5.7 \%$, $n=11 / 193)$ were SPs and the remainder were GDPs (94.3\%, $n=182$ ). A wide distribution was found in relation to age and number of years of experience. All South African provinces were represented.

\section{Sterilisation methods used for endodontic files}

Only two sterilisation methods were reportedly used for the reprocessing of endodontic files. More than threequarters of the respondents $(76.6 \%, n=141 / 184)$ indicated sterilising files using a steam autoclave.

Cold sterilant solutions were used by almost one-quarter of the respondents $(23.4 \%, n=43 / 184)$. Nine respondents practiced single-use of endodontic files and were excluded from analysis as they did not sterilise and reprocess files.

\section{Sterilisation before first use}

With regards to initial sterilisation procedures (i.e. when removing endodontic files from the manufacturer's packaging), the majority of respondents $(71.5 \%, n=139 / 193)$ indicated that they did not sterilise endodontic files prior to first patient use. The remaining 28.5\% $(n=55 / 193)$ indicated completing a pre-use sterilisation cycle.

\section{Sterilisation before re-use}

Almost all the respondents who reused their endodontic files $(97,2 \%, n=176 / 181)$ indicated routinely sterilising the files prior to re-use on subsequent cases. A small number $(2,8 \%, n=5 / 181)$ indicated the opposite.

Twelve respondents $(n=12 / 193)$ did not answer this question as they practiced either single-use of their files or did not sterilise endodontic files (or a combination of these reasons).

\section{Single-use of endodontic hand files}

Only $10.9 \%$ ( $n=20 / 184)$ of respondents reported practising single-use of endodontic hand files. The remaining $89.1 \%(n=164 / 184)$ reused hand files on multiple cases following reprocessing procedures. Nine respondents did not answer this question as they indicated not using hand files routinely.

\section{Single-use of engine-driven files}

Rotary and reciprocating files were reprocessed and reused on multiple cases by the majority of respondents (81.5\%, n=150/184).

The remaining respondents $(18.5 \%, n=34 / 184)$ indicated practicing single-use of these instruments. Nine respondents did not answer this question as they did not use engine-driven files routinely.

\section{DISCUSSION}

Although the overall response rate of this survey was low, the response rate was in line with previous South African survey findings conducted on similar cohorts. ${ }^{9}$ Furthermore, the survey was completed by respondents from all nine South African provinces and valuable information was therefore collected from a wide geographical distribution. The responses of prosthodontists were included in the present study as South Africa does not train specialist endodontists. ${ }^{10}$ 
A previous study reported that the single-use of endodontic files had not been adopted by any general dental practitioners surveyed in one major South African city. The results of the present study, which included both a greater number and wider distribution of South African dentists, are in disagreement with the previous findings. This finding highlights the importance of obtaining representative sample sizes, and cautions against the extrapolation of the results of smaller scientific investigations to a broader population.

The philosophy regarding single-use of endodontic instruments originated in the United Kingdom in response to concerns surrounding the potential spread of prion disease ${ }^{11}$ as a result of the inability to adequately clean and sterilise endodontic files and reamers. ${ }^{2}$ This view was however not universally shared. ${ }^{5}$ Whilst several benefits of a single-use approach exist, such as a reduced risk of file separation ${ }^{12}$ and no risk of cross contamination ${ }^{2}$, the reprocessing and reuse of endodontic files will likely continue in many countries due to the increased cost associated with single-use protocols of endodontic instruments. ${ }^{8}$

When any dental instruments are to be reused, they must be both thoroughly cleaned of bioburden and sterilised to prevent cross-contamination between patients. Although sterilisation may be achieved by several different methods, semi-critical and critical instruments - such as endodontic files - should be sterilised by autoclave. ${ }^{13}$ When considering multiple or single-use of any dental instrument, manufacturer recommendations in conjunction with ISO 17664:2017 and local regulatory guidelines should be followed at all times. ${ }^{2}$

Previous investigations have demonstrated that endodontic files and burs are not sterile at the time of purchase and that sterilisation should be performed prior to first use. ${ }^{4}$ Less than one-third of the respondents to the present survey complied with this recommendation.

In recent times, however, manufacturers have created pre-sterilised, single-use endodontic instruments which do not need to be sterilised prior to initial use. ${ }^{14}$ It is unknown how widespread the use of such pre-sterilised endodontic files are, but this possibility may have contributed to the high number of respondents who indicated not sterilising their files prior to initial use. Future studies may investigate to provide clarity on this issue.

The finding that nearly one quarter of respondents continue to use cold sterilisation as the sole means of reprocessing endodontic files was disappointing. Placement of endodontic files in cold sterilant solutions, such as glutaraldehyde, has been demonstrated to be inferior to steam methods and is no longer recommended as a primary means for the sterilisation of endodontic files. ${ }^{13}$

Additionally, it has been demonstrated that cold sterilising solutions, such as glutaraldehyde, may take up to ten hours to sterilise an instrument. ${ }^{15}$ The finding that some respondents of the present study did not sterilise endodontic files at all before reuse was alarming. It is however possible that these respondents misunderstood the question.

\section{CONCLUSION}

Within the limitations of this study, the majority of South African GDPs and SPs were demonstrated to reuse endodontic files on multiple clinical cases following reprocessing procedures. A large number of respondents used acceptable methods of sterilisation for reprocessing endodontic files. A significant proportion however continue to use unacceptable methods such as cold sterilisation with glutaraldehyde solution.

Whilst only a limited number of South African dental practitioners have adopted the routine practice of singleuse of both engine-driven and hand endodontic files, this number is higher than previously reported.

\section{Declaration}

The Author(s) declare that there is no conflict of interest.

\section{Funding}

This research did not receive any specific grant from funding agencies in the public, commercial, or not-forprofit sectors.

\section{References}

1. Carrotte P. Endodontics: Part 5. Basic instruments and materials for root canal treatment. Br Dent J. 2004; 197(8): 45-64.

2. Walker JT, Dickinson J, Sutton JM, Raven NDH, Marsh PD. Cleanability of dental instruments - implications of residual protein and risks from Creutzfeldt-Jakob disease. Br Dent J. 2007; 203(7): $395-401$.

3. Letters S, Smith A, McHugh S, Bagg J. A study of visual and blood contamination on reprocessed endodontic files from general dental practice. Br Dent J. 2005; 199(8): 522 - 25.

4. Morrison A, Conrod S. Dental burs and endodontic files: are routine sterilization procedures effective? J Can Dent Assoc. 2009; 75(1): 39.

5. Messer H, Parashos P, Moule A. Should Endodontic Files Be Single-Use Only? A Position Paper From The Australian And New Zealand Academy of Endodontists. Aust Endod J. 2003; 29(3): 143-5.

6. Mehtar S, Shisana O, Mosala T, Dunbar R. Infection control practices in public dental care services: findings from one South African Province. J Hosp Infect. 2007; 66(1): 65-70.

7. Buchanan GD, Warren N, Gamieldien MY. Debris contamination of endodontic hand files in dental practice. S Afr Dent J. 2018; 73(6): 440-4.

8. Buchanan GD, Warren N. Single-use of endodontic hand files: perceptions and practise. J Infect Prev. 2019; 20(1): 32-6.

9. Snyman L, van der Berg-Cloete S, White J. The perceptions of South African dentists on strategic management to ensure a viable dental practice. S Afr Dent J. 2016; 71(1): 12-8.

10. Fernandes NA, Herbst D, Postma TC, Bunn BK. The prevalence of second canals in the mesiobuccal root of maxillary molars: A cone beam computed tomography study. Aust Endod J. 2018; 1 -5.

11. Head MW, Ritchie D, McLoughlin V, Ironside JW. Investigation of PrPres in dental tissues in variant CJD. Br Dent J. 2003; 195(6): 339 - 43.

12. Yared G. In vitro study of the torsional properties of new and used profile nickel titanium rotary files. J Endod 2004; 30(6): 410-2.

13. Hurtt CA, Rossman LE. The sterilization of endodontic hand files. J Endod. 1996; 22(6): 321-2.

14. Webber J. Shaping canals with confidence: WaveOne Gold single-file recipocating system. Roots. 2015; 1(3): 34 - 40.

15. Özalp N, Ökte Z, Özcelik B. The Rapid Sterilization of GuttaPercha Cones with Sodium Hypochlorite and Glutaraldehyde. J Endod. 2006; 32(12): 1202-4. 